\title{
Crowdsourcing System using Blockchain Technology
}

\author{
Neha More, Dilip Motwani
}

\begin{abstract}
Crowdsourcing frameworks have been receiving a lot of enthusiastic acceptance these days, and there has been a rise in significant raise in concern as well. Crowdsourcing frameworks are excellent tools that can coordinate the human insights of specific instances, and organizations together globally and help comprehend and collaborate intricate chore. Notwithstanding, these central structures subject to the inadequacies of the trusted standard like standard financial foundations. For example, single motivation behind dissatisfaction, higher organizations cost, and security disclosure. An idea is to use a shared structure for crowdsourcing basis blockchain, wherein the work of the requester is directed by the swarm of workers without relying on central freely supporting systems or foretold customers that are inclined to organizations with enrolling certifiable characters. In thought, the proposed framework design would permit Users to enroll, post, or get an endeavor securely. The design would likewise furnish clients with undeniable degree of protection, and security, and the proposed system additionally has low administration costs. By extending the adaptability and adaptability of publicly supporting the reason for existing is to show the crowdsourcing logic with intelligent contract. As per this structure, a requester needs to deposit the venture money, while posting task. The stored deposited amount is escrowed with the framework, and on the concurred task fruition, the laborer will get the undertaking amount from the escrow. Because of this system, a requester won't need to pay more than what an assignment merits, as indicated by a rule unveiled when errand is posted; and every laborer will unquestionably get an installment dependent on the rules.
\end{abstract}

Keywords: Blockchain, cryptography, Escro

\section{INTRODUCTION}

$S_{\text {to }}$ crowdsourcing. Crowdsourcing demonstrates to an unbelievable arrangement approach, whenever used in the ideal spot at the convenient time. Merriam-Webster characterizes crowdsourcing in the process of "the act of gaining desired services, thoughts, or substance by requesting commitments from an enormous gathering of individuals and particularly from the online local area instead of from traditional workers or suppliers" [5], and the word can be named a portmanteau word, with its roots in the words' "crowd" and "outsourcing".

Manuscript received on September 20, 2021.

Revised Manuscript received on September 25, 2021.

Manuscript published on September 30, 2021.

* Correspondence Author

Ms. Neha More*, Department of Lecturer, Shri Bhagubhai Mafatlal Polytechnic in Information Technology, Mumbai. (Maharashtra), India. E-mail. Neha.more@sbmp.ac.in

Dr. Dilip Motwani, Associate Professor, Vidyalankar Institute of Technology, Mumbai. (Maharashtra), India. E-mail. dilip.motwani@vit.edu.in

(c) The Authors. Published by Blue Eyes Intelligence Engineering and Sciences Publication (BEIESP). This is an open access article under the CC BY-NC-ND license (http://creativecommons.org/licenses/by-nc-nd/4.0/) has been riding the influx of fame and eagerness around

Most of the contributors in a crowd sourced project is either paid freelancers, or people getting involved pro-bono, or a combination of both. Ina crowdsourcing model organizations can subcontract work to individuals or other organizations, can work or open anywhere in the nation or around the globe. Such a flexible structure of workflow allows organizations tap into the huge scope of aptitudes and skill without gaining the regular overhead costs of internal delegates/workers. The human-centric crowdsourcing models involve three major roles: requesters, workers, and a connected crowdsourcing affiliation. Requesters endeavor that pursue PCs yet straightforward for an individual to acknowledge by means of the crowdsourcing system. A gaggle of employees/workers curious about this task competes and present results to the crowdsourcing system, while the petitioners will then shortlist a correct and fool-proof solution (that solves the task) and provides the rewards to the worker [8]. Crowdsourcing system can be the future way for all sorts of businesses to accumulate new assets, investors, and even ideas. The system can have some weakness which can pose certain challenges. Some of the challenges can be listed as under:

- Maximum crowdsourcing associations run their commerce on a unified server. This server encounters from a single point of breakdown, naturally [5].

- Most of the Crowdsourcing frameworks are uncovered to Distributed Denial of Service attacks that make the services inaccessible [8][1].

- Confidentiality can be a pressing challenge- When moving toward people for their responses, an organization may have to disclose delicate information or data to find a solution which will truly work [5]. For instance, in case an organization is pursuing responses to an issue like 'item bundling' then this can be an indication that there is some inadequacy in the organization's current bundling frameworks [12]. An extend might not be applicable for crowdsourcing in-case, it relies on monetarily touchy data. We propose a distributed structure for crowdsourcing which is blockchain-based. It has several benefits like progressing user security and repair basic utilize, moving forward the flexibility of crowdsourcing with low value. In this manner, our model has the capability to intrude on the typical model of crowdsourcing [11] [3]. Ordinary crowdsourcing relies upon the united structure for posting and getting errands, it is basically, a three-party system model. The blockchain development has inured an encouraging strategy to enlightening the trust issues among various social occasions [3].

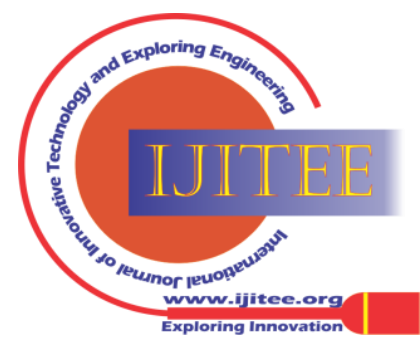




\section{Crowdsourcing System using Blockchain Technology}

Crowdsourcing endeavors are represented as "programs" running inside the blockchain, including task posting, task getting, arrangement accommodation, and prize undertaking. Astoundingly, so on get pleasant results, the requester simply allows qualified workers who turn up at the exceptionally minimum reputation impetus to ask the endeavor.

\section{TRADITIONAL CROWDSOURCING SYSTEM:}

The Commonplace model of crowdsourcing brace with coordinates plan with "centralized" by and large recommending to the venture satisfying of strategy which could be a fundamental piece of the ordinary crowdsourcing system [1]. The centralized crowdsourcing models accomplishes the use of forthrightness in both building up a software application and managing the framework [8]. Anyhow, any brought forth course of action goes with the single reason of disillusionment weakness, with the solitary factor being the main position or director of the arrange within the crowdsourcing worldview, as attacking them makes the organization gets difficult to reach.

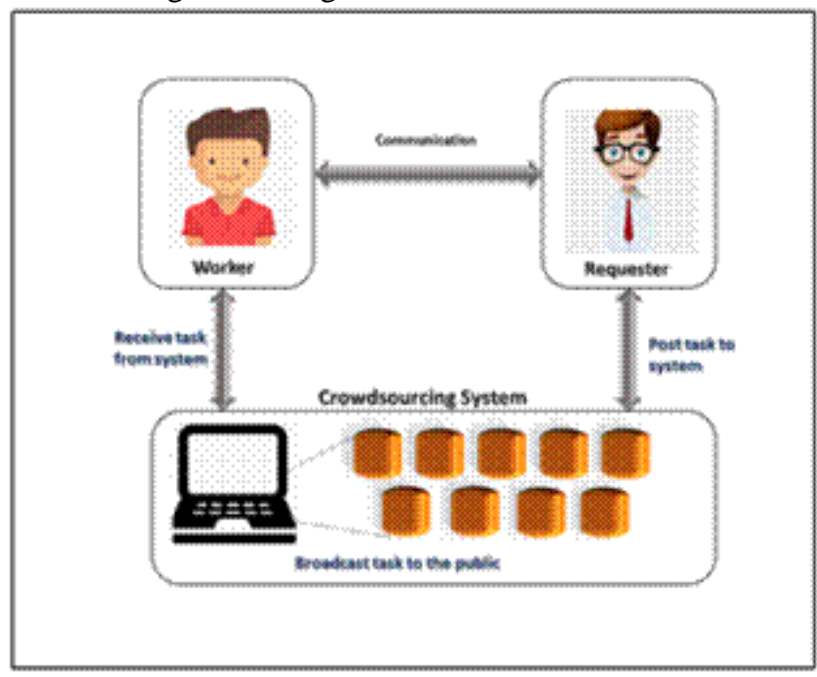

Fig.1-Traditional Crowdsourcing System

Fig.1. illustrates the traditional system of crowdsourcing. In which Requesters yield errands which are attempting for PCs in any case smooth for human to total through the crowdsourcing system [8]. A part of laborers who are enthusiastic on this task fight and yield answers of the task for the crowdsourcing system, whereas requesters want to select an authentic course of action (ordinarily the finest or to begin with one that secure the errand) and grant the relating laborers the cherish. In any case, in spite of the thriving of the crowdsourcing systems, they are subordinate upon the deficiencies of standard show, which realizes a few inevitable troubles, for example, frameworks are feeble against DDoS ambushes. Typically, since they are brought together server chances of single point dissatisfaction and nonappearance of security.

\section{LITERATURE SURVEY}

[1] "Toward a Blockchain Enabled Crowdsourcing Platform"- Dimitrios G. Kogias and Helen C. Leligou Published by the IEEE Computer Society 11 September 2019, IEEE)
Paper explains Crowdsourcing to explore, discuss, and visualize various ways to use for various purposes. The purpose would include different fields such as combining data, gathering assets and social event workers to implement assignments of various sizes with various objectives. Information uprightness and non-repudiation are of most extreme significance in these frameworks and are not even ensured. Blockchain innovation has been demonstrated to upgrade these perspectives. During this study, research that the Blockchain innovation will introduce crowdsourcing frameworks. This offers real-life crowdsourcing examples and investigates the advantages of utilizing Blockchain, fundamentally as a database system.

[2] "On Enabling Machine Learning Tasks atop Public Blockchains: A Crowdsourcing Appro®ach”- Yuan Lu, Qiang T, Guiling W.2018 IEEE International Conference on Data Mining Workshops (ICDMW)

This research presents the current and developed blockchain with a specifically good agreement and innovation. This proposition deals with an alluring open door for decentralized sharing economy. AI is routinely one important subroutine in decentralized biological system. AI programs are difficult and typically, random for exploring and creating best results. They fall under the natural components of blockchain, where hidden hubs can be used as-a-whole to execute such mind boggling and randomized projects. Given similarly the constraints of extant valid figuring frameworks, we recommend a publicly supporting idea from the inclined hypothetical perspective to decide the pressure. Altogether, we plan a basic inspirational instrument that the execution of a nice extent of difficult undertakings are consistently up-held openly by methods of the blockchain, and the result might be redirected. Especially, our show works inside the circumstances that there is no believed intact; moreover, it does not simply work inside the conventional model of non-scheming expert associations, still can similarly bear any likely coalition up to $n-1$, where ' $n$ ' is that the total number of expert centers. It also shows the best way to deal with use to publicly supporting two regular kinds of AI tasks through open blockchain. This assumes and accepts that our response is encouraging to dispatch the decentralized applications having the good extent of AI programs. [3] "Vizsafe: The Decentralized Crowdsourcing Safety Network"- Peter A. Mottur 2018 IEEE

A resourceful city utilizes exploitation through propel transporting of constitution to inhabitants as well as augmentation tenant's own fulfilment. To manage open assistance transport a gigantic show of sensing element and Iota appliance will be shipped off satisfy the data necessities of a sensible way, and any city's biggest and coordination compound sensor are used to organize and cash register and take cognizance of the state of its residents. Fixture substance abuser of the city's framework and presidential term can report any outcome as and when required. The most obvious reason that forestalls an enormous part of the people from doing so is first matter, the absence of a phase angle with the trade goodness channels.

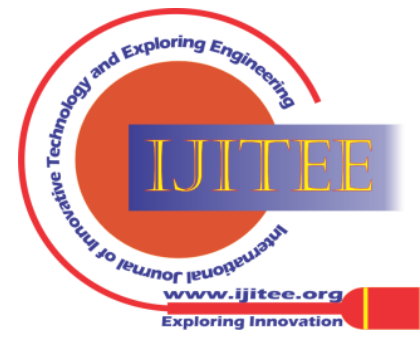


Also, the insufficiency of brief inspirations - as-a-general rule, an issue would be someone else's business to oversee.

To address such military issues, the Vizsafe stage is proposed to set-up the pack and persuade customers or the framework to introduce information that is valuable and lay everyone at an incredible benefit. This study is taken care of on a decentralized record, through the workplace of blockchain, utilizing splendid arrangements, SPOT tokens are used as an arousal power to individuals that moves event accounts, and this is paying little heed to the way that whether it is a potential security risk or an imperfect tense institution. It is the slight push that 'my possibility' from makes 'someone else's anxiety'. At the point when metropolitan regions Menachem begin demonstrating totality or focused help, people quit being customers and fix customers, rather they Begin to contribute information, which increments and engages their total benefit, while reducing costs for associations and fix providers. They change to being moral force associates in holding up the organization during the time they stay as well as work.

[4] Efficient "ZebraLancer: Private and Anonymous Crowdsourcing System atop Open Blockchain"- Yuan Lu, Qiang Tang, Guiling Wang.2018 IEEE 38th International Conference on Distributed Computing Systems"

The study centers around the system plan of the first harmonic challenges of detelephone exchange izing, publicly supporting, that incorporates data spill and character break. The underlying advances incorporate our re-suitable and afterward exhibit how a framework stage settles variant among blockchain straightforwardness and data confidentiality, which is an essential use case for publicly supporting. ZebraLancer ensures: (i) a requester will not compensate data deservingness, consistent with a course of action detailed when his/her task is disseminated through the blockchain. (ii) Each expert in-actuality gets a portion maintaining the methodology in-case he/she submits data to the blockchain. (iii) the properties above are recognized without any exchange referee, still furthermore outwardly delivering any information to the open blockchain. They will behead our show for a banner image remark task and pass it on at the time of the tryout net of Ethereum. The fittingness of our show in real world blockchain can be set up by the examination.

[5]"zkCrowd: A Hybrid Blockchain-based Crowdsourcing Platform"- S. Zhu, Z. Cai, H. Hu, Y. Li and W. Li, IEEE Transactions on Industrial Informatics.doi: 10.1109

An encouraging decentralized worldview - Blockchain, can be solely executed to beatnik the deficiency of the common publicly supporting systems, yet additionally to acquire particular turns of events, like decentralization and obligation the board. Some necessity synonyms of noun things or fundamental limitations of blockchain are so often tended to record as a hard copy, when it is united in publicly supporting, and that can fruit the introduction bottleneck inside the publicly supporting systems. During this paper, a farther favored place of the prevalence of mixture blockchain and publicly supporting, we propose an inventive creamer pitcher blockchain publicly supporting stage, named zkCrowd. The voltage gains of a blended blockchain anatomical structure, splendid arrangement, twofold records, and twofold accord shows are interplayed and coordinated to guarantee symmetricalness, check trades, and defend security. Both, the theoretical assessments, and exams are performed to survey benefits of zkCrowd over the best in class.

\section{RELATED WORK}

We deliberately examined pertinent works and momentarily mulled over on lack of the new arranged solutions. Numerous crowdsourcing organizations are planned and created using a centralized architecture [7], [15], [14]. The role of crowdsourcing that incorporates broadcasting an assignment from requester, applying for an assignment, choice of the laborer, and the bodily structure of payment are kept up by the centralized crowdsourcing frameworks. Up-work [14] and Consultant [7] are extraordinary crowdsourcing stages allowing requester to adroitly enroll laborers to induce the assignment courses of action. In any case, they would need user's positive details and store client information, assignment details within assembled organize that might encounter sick impacts of assurance spillages, DDoS, and the effect of single reason of dissatisfaction. Some of the researches on the distributed crowdsourcing system include the following: S. Zhang, et al [2] presented a protocol to purpose distributed crowdsourcing system in Delay-tolerant networking (DTN). The divine planning through a computation errand is to attain the negligible shuffle pan. p.yang,et al[6] prescribed an errand dispersion strategy by utilizing mixer relationship in crowdsourcing framework Whereas [2] and [6] co-ordinate on assignment satisfaction in a dispersed way, they spring benefit through centralized framework. Our design is to construct the decentralized crowdsourcing framework. Our modern font primordial can fall out these insufficiencies by employing a blockchain-based decentralized system with much broader targets. This incorporates storing of all encrypted transactions on blockchain as database and payment prepare is computerized from loading cash into the escrow to remembering the payment from the escrow after deducting entry mission.

\section{Blockchain:}

The companion - peer network is the square chain arranged that is situated at the highest point of the web. This square chain may be named on the grounds that the basic innovation, bringing about the upset of the commerce and accordingly the public authority. It is a public electronic record worked around a distributed framework and will be straightforwardly divided between dissimilar clients. This is for an immutable note of exchanges, so every single will be connected through the previous sole. At whatever point another exchange is added, recently added information turns into another square inside the chain. [13]. The definition of how multiple copies of such blocks are often constructed and maintained during a distributed fashion, is often defined by the underlying Blockchain protocol. The blockchain uses a ledger, it is a digital file that keep tracks all transactions. The ledger file is distributed over the planet via a network of pcs. Which are responsible for storing data and executing computations. Each and every computer represents a "node" of the blockchain network and features a replica of the ledger file. Each node within the blockchain is keeping a replica of the ledger. 


\section{Crowdsourcing System using Blockchain Technology}

The ledger only keeps track of each transaction, and does not keep track of balances. The digital pieces of data creates the "Blocks" on the blockchain. The three parts of the mandatory information are (i) Squares- it stores the data about all exchanges such as date, time. (ii) Blocks-it store data of those who are taking part in transactions. (iii) Blocks store the data which identify them from different squares.

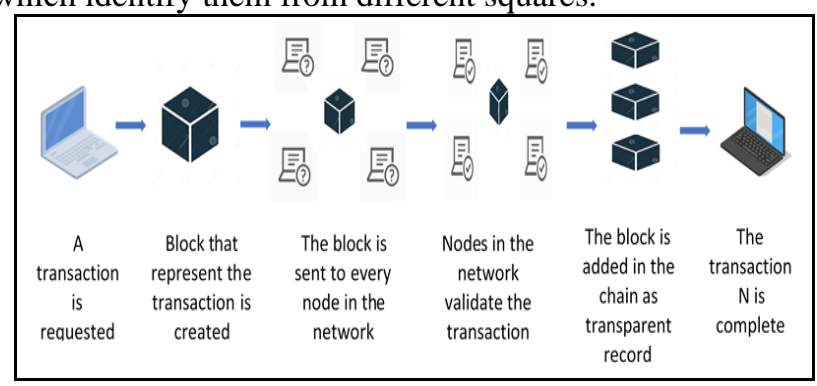

Fig.2 blockchain process

Fig. 2. Illustrates the blockchain process [13]. In this process as soon as the trade is cited, a data structure for maintaining the plan of trades is passed onto all centers or any center within the framework. Every one of the centers affirm the squares prior to addition of anything to the blockchain. At a point, centers perform square check and will bring corroboration in respect to job. Subsequently, every new center within apposite plan about blockchain which contains entire replica. Right while any other square is formed, it is shipped off each center point within the framework of blockchain. Around by then, each center asserts the square and checks whether or not the information imparted there's right. In the case if all is proper, square will be augmented with blockchain within every center.

\section{SHA 256:}

National Institute of Standards \& Technology has invented a unique algorithm called as SHA - Secure Hash Algorithm to avail secure hash bits. Nowadays, hashing is executed using their latest version i.e. SHA-256. The key procedure is inured on the name of this algorithm and proportionately, the algorithm calculates the hash length of 256 bits which is very remarkable [10]. The rule behind this is the input value added to the end output that transforms the encryption algorithm into "hashing" algorithm, the building piece of a standard hash function. The underlying cypher block has 64 rounds and hence, a 2048-bits expanded internal key (64 x 32 bits) is accessed from the block of message which is to be compressed. At the input, the key has 512 bits which is expanded four times to form 2048-bits internal key for our cypher block. This SHA-256 algorithm is divided into two stages as below: 'pre-processing' \& 'hash computation' [10]. Here, pre-processing involves padding a message and parsing the padded message into m-blocks. Initialization values are set and is to be used in the hash computation. Hash computation produces a message plan from the cushioned message. The yield hash esteem produced by this hash computation is used to decide the message digest. Hash computation includes message plans, functions, capacities, and word activities that are created iteratively to get hash esteem. The security of SHA-256 hash calculation is based on the amount of the hash values.

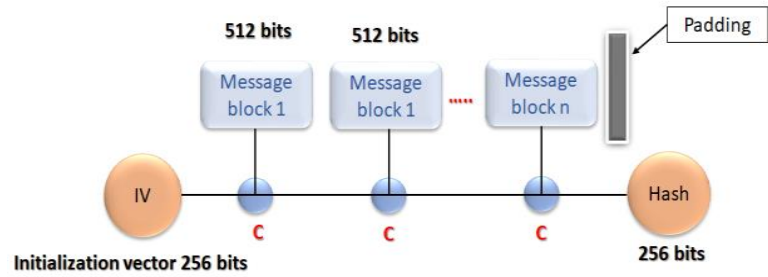

Fig.3 Algorithm of SHA256

\section{PROPOSED SYSTEM}

For directing diverse crowdsourcing [4] [8] advances, proposed protocol based on blockchain [16]. A blockchain-based protocol too gives a promising bearing to impact the reputation of the system. In this proposed system, the crowdsourcing permits each single step of crowdsourcing strategy to be managed as exchanges in Blockchain. This would offer assistance make superior certainty inside the organize clients, as well as address different sorts in regard to conceivable assaults for a crowdsourcing stage which is brought together.

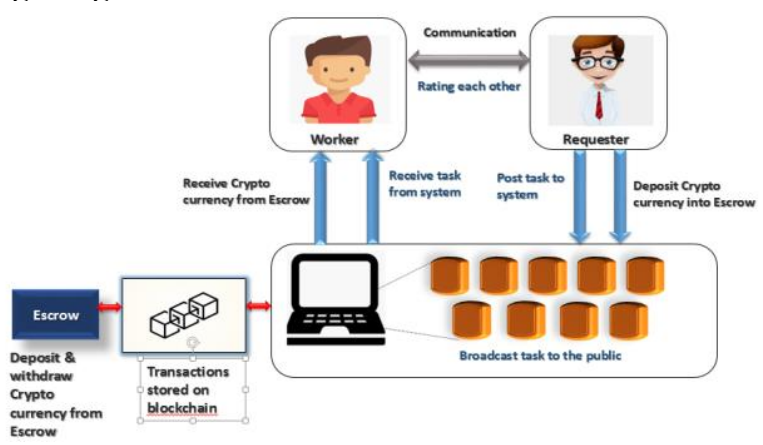

Fig.4 Crowdsourcing system using Blockchain

Fig. 4. A visual representation of the suggested crowdsourcing structure based on blockchain [8]. Within the provided arrangement, requester and worker are two sort of handlers. Note that there's no fundamental server, also crowdsourcing system supports the Turing-complete ventures. This portrays, strategy of crowdsourcing [8]. Both the handlers should compulsory enroll for getting their capabilities prior then receiving their controls from the system.

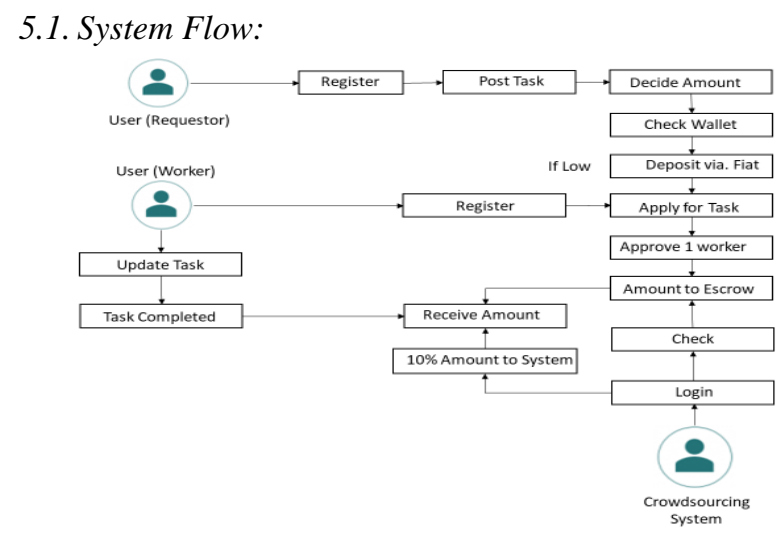

Fig.5 System Flow

Published By:

Blue Eyes Intelligence Engineering and Sciences Publication

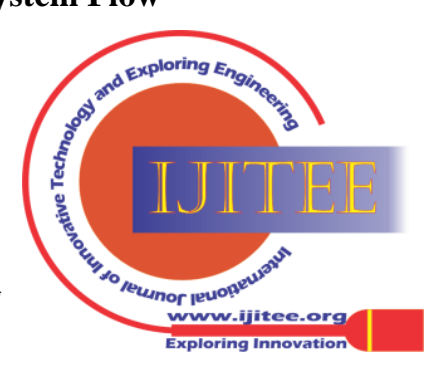


Fig.5.Characterizes the system work flow containing Requester, Worker, and Admin as 'Modules'. The work flow of the proposed system alongside modules is as below:

- User can be either a requester or a worker. However, a requester who wishes to finish the task or a worker who wants to apply for a job must register first before login into the system.

- Requester can load a task with characterized amount and time frame/due dates on decentralized crowdsourcing system.

- Requester looks over the wallet for balances and deposits the amount in the form of fiat currency if less balance on wallet through blockchain.

- The requester will pay $10 \%$ of the amount to the decentralized crowdsourcing system. This will be useful winning to the system and covered up from the requester, this amount can be utilized for offering remunerate to the fresh requester/ Worker.

-Depending on the skills and capabilities numerous workers can apply for the errands.

-Requester can choose any specialist or different Workers depending on their profile and allot an errand.

-When the errand is allocated, Requestor is expected to utilize the Virtual Crypto-currency in the Escrow Account that has been overseen by a crowdsourcing system in-order to down pay the errand amount.

-Requestor can purchase the crypto-currency using Fiat Currency.

-The crypto-currency, including all exchange points of interest, incorporate a few of the taking after, deposit amount in the wallet, in escrow, and in workers' wallets stored on the blockchain.

-Worker can submit the errand to the requester in percentage or module wise. Worker in-turn will earn errand wise pre-decided sum through crypto-currency from the escrow.

- Once the errand is completed successfully, Worker's account will be loaded with the pre-endorsed escrowed amount.

Requester and Worker both can allow rating upon the basis of the work and errand executed individually towards long term utilize.

Below are the accompanying Modules of the system:

1.Requester: At beginning, the requester must register to login into system. Post that, below exercises need to be executed by the requester:

-Placing errand with characterized amount and time frame/due dates on decentralized crowdsourcing system.

- Check for the amount in wallet and deposit the amount if less balance in the wallet.

- Choose any specialist or different Workers depending on their profile and allot an errand.

-Acknowledge an errand after consummation and rate the specialist as per their work.

2.Worker: At the beginning, the worker must register and login into the system. Post that, below exercises need to be executed by the worker:

-Choose an errand as per ability and apply for the task.

-Deals with errand after endorsement. - Receive amount on fruitful culmination and give a rating to the requester dependent on their acceptance and response.

3.Crowdsourcing system: The crowdsourcing system which is responsible for keeping up the escrow has to login itself. This full process is computerized starting from getting
- Finishes the errand and present the errand to the requester

amount within the escrow to relieving amount out of the escrow post subtracting the charges of portal.

\subsection{Snapshots Of The Proposed System}

\section{$>$ User register}
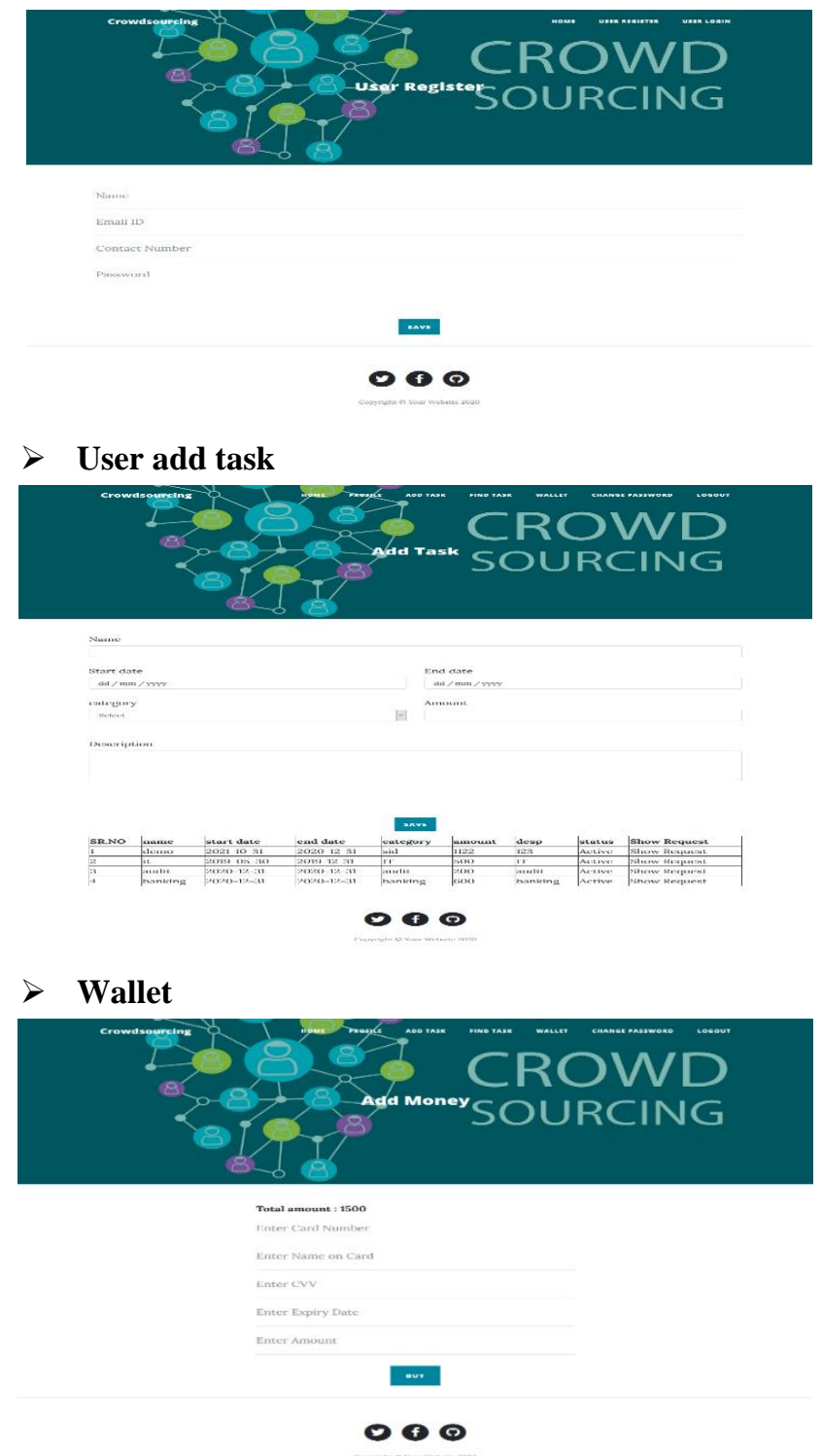

VI. RESULT ANALYSIS

As the peculiar properties of this framework, it has been confirmed that a decentralized Crowdsourcing system delivers much preferable execution over a centralized system. The proposed framework prevents from the blockage problem and gives sensible exchange between the data and the prize. Decentralized crowdsourcing, along escrow framework gives secure and safe cash routing between parties as well as it allows each and every transaction according to party's requirement. This framework contrasts and customary framework which follows the incorporated path under different execution variables to approve the achievability of the model for genuine ease of use situations. Fig 6 . below shows the analysis of comparison between centralized Crowdsourcing system and decentralized system.

Published By:

Blue Eyes Intelligence Engineering and Sciences Publication 


\section{Crowdsourcing System using Blockchain Technology}

Table 1. Comparison of Crowdsourcing with ESCROW and Crowdsourcing without ESCROW.

\begin{tabular}{|c|c|c|}
\hline Factors & $\begin{array}{c}\text { Crowdsourcing with } \\
\text { Escrow }\end{array}$ & $\begin{array}{c}\text { Crowdsourcing } \\
\text { without Escrow }\end{array}$ \\
\hline Task Processing & 8 & 3 \\
\hline Task Redundancy & 3 & 8 \\
\hline Task Efficiency & 8 & 3.5 \\
\hline System Usability & 7 & 4 \\
\hline System Security & 8.5 & 3 \\
\hline
\end{tabular}

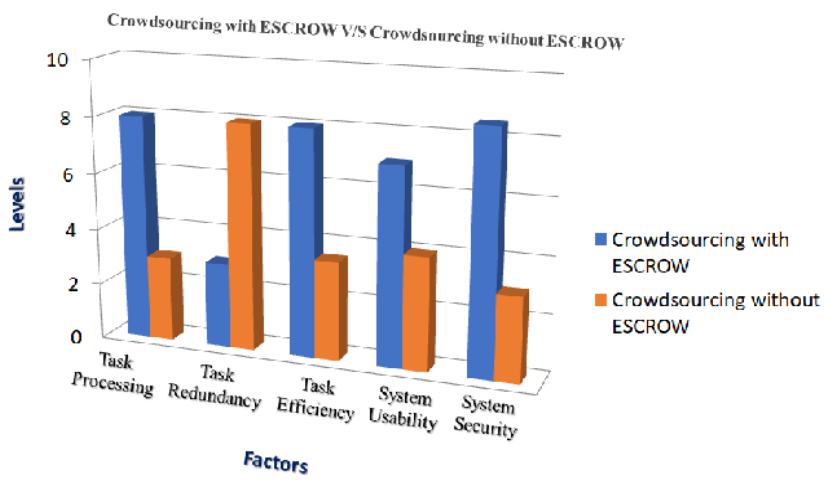

Fig. 6. Crowdsourcing System (With ESCROW v/s Without ESCROW)

\section{CONCLUSION}

The paper presented design of blockchain basis crowdsourcing. The paper also analyzed the traditional centralized crowdsourcing system and presents the drawbacks of such a system suffers from privacy disclosure, if failed then entire system fails, tremendous maintenance fee, et al. An advanced blockchain based crowdsourcing system can handle the shortcomings of crowdsourcing frameworks by boosting their allure to fathom a few issues and extending the potential of their application. A Blockchain database holds the outright, permanent, and permanent history of all the exchanges, resources, valuables, and instructions executed as the absolute initial one. Blockchain too permits partaking parties - and only those parties - to share reachable, transparent, and reliable information. Embracing Blockchain technology, the ledger of all transactions can be kept in a set of nodes (having a place either for workers or for requesters), precluding a central authority's/entity's requirement. The proposed system centers on web-enabled applications because of its straightforwardness it'll somewhat influence the speed of operations of the entire crowdsourcing process. This is often the restriction of the framework; subsequently, implementing mobile-based application is the advantageous and future-ready arrangement.

\section{REFERENCES}

1. "Toward a Blockchain Enabled Crowdsourcing Platform"- Dimitrios G. Kogias and Helen C. Leligou Published by the IEEE Computer Society 11 September 2019, IEEE)

2. "Minimum makespan workload dissemination in dtns: Making full utilization of computational surplus around,"- S. Zhang, J. Wu, and S Lu.in Proceedings of the fourteenth ACM international symposium on Mobile ad hoc networking and computing. ACM, 2013, pp. 293-296

3. "Vizsafe: The Decentralized Crowdsourcing Safety Network"- Peter A. Mottur 2018 IEEE

4. "Zebra Lancer: Private and Anonymous Crowdsourcing System atop Open Blockchain"- Yuan Lu, Qiang Tang, Guiling Wang.2018 IEEE 38th International Conference on Distributed Computing Systems"

5. "zkCrowd: A Hybrid Blockchain-based Crowdsourcing Platform"- S. Zhu, Z. Cai, H. Hu, Y. Li and W. Li, IEEE Transactions on Industrial Informatics.doi: 10.1109

6. "friend is treasure: Exploring and exploiting mobile social contacts for efficient task offloading"- P. Yang, Q. Li, Y. Yan, X.-Y. Li, Y. Xiong, B. Wang, and X. Sun, IEEE Transactions on Vehicular Technology, vol. 65, no. 7, pp. 5485-5496, 2016.

7. “Freelancer", https://www.freelancer.com, [online]

8. "A Blockchain-enabled Trustless Crowd-Intelligence Ecosystem on Mobile Edge Computing”- Jinliang Xu, Shangguang Wang-2019.

9. "CrowdBC: A Blockchain-based Decentralized Framework for Crowdsourcing"-Ming Li, Jian Weng, Anjia Yang, Wei Lu,Yue Zhang, Lin Hou, Jia-Nan Liu, Yang Xiang, Robert H. Deng

10. "Application-Oriented SHA-256 Hardware Design for Low-Cost RFID”- Xiaolin Cao, Maire O’NeillCao,2012 IEEE International Symposium on Circuits and Systems. doi:10.1109/iscas.2012.6271509

11. “A Proof-of-Trust Consensus Protocol for Enhancing Accountability in Crowdsourcing Services"- J. Zou, B. Ye, L. Qu, Y. Wang, M. A Orgun and L. Li, IEEE Transactions on Services Computing, vol. 12 , no. 3, pp. 429-445, 1 May-June 2019.

12. "Blockchain-based Credit and Arbitration Mechanisms in Crowdsourcing"- Y. Ding, Z. Chen, F. Lin and C. Tang, 2019 3rd International Symposium on Autonomous Systems (ISAS), Shanghai, China, 2019, pp. 490-495.

13. A Survey of Blockchain Enabled Cyber-Physical Systems"-Heena Rathore,Amr Mohamed and Mohsen guizani.

14. upwork", https://www.upwork.com, [Online]. Truelancer", https://www.truelancer.com, [Online].

15. Ethereum. Jun. 2019. [Online]. Available: https://www ethereum.orgACM, New York, NY, USA

\section{AUTHORS PROFILE}

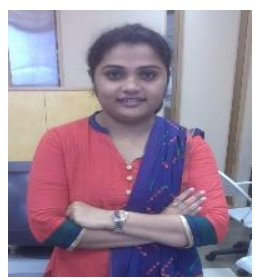

Ms. Neha More, Department of. Lecturer, IT SVKM's SBMP, Mumbai Neha.more@sbmp.ac.in Information technology helps to build and grow the commerce and business sector and generate maximum possible output. The time taken by different sectors to generate business is now minimized with an advancement in Information technology. After completion of my Engineering in 2008, I was placed in multinational Company. Afte acquiring some skillsets, I switched my profile into teaching and joined as a Lecturer in government aided diploma engineering college, viz. Shri Bhagubhai Mafatlal Polytechnic in Information Technology department. Utilizing my interest in IT I taught the subjects like System Programming, Operating System, Web technology, Internet of Things. Also, I got admitted for masters in Computer Engineering in Vidyalankar Institute. Moderately developed interest in Crypto currency which led me research in the field of Blockchain.

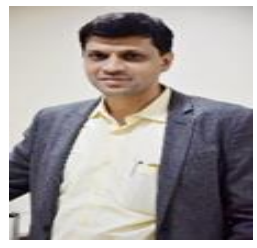

Dr. Dilip Motwani, Associate Professor, Vidyalankar Institute of Technology, Mumbai. (Maharashtra), India. dilip.motwani@vit.edu.in I consider myself to be fortunate in taking a quantum leap into the field of Computer Engineering when it had begun to make an impact on the lives of people across the globe. I joined as a lecturer in an engineering college and started my work with complete hands on approach in designing and implementing computer network for all the laboratories in the college and it turned out to be a great learning experience. Completion of my post-graduation (ME) in Information Technology equipped me with a new skillset in Network Technologies and Mobile Computing through my project work entitled "Bandwidth Management". While utilizing all my computer network skills I also learnt open source Linux operating system environment and mastered Cisco technology. Introduction to the ISP (Internet Service Provider) atmosphere further empowered my functionality in the computer networking area involving gateway configuration and installation of routers, LAN Linux Server, etc. During the period, I got opportunity to work with organizations like CDAC and IGNOU as their program coordinator which also helped me in enhancing technical skills to great extent. My 21 year old association has made me acknowledge the reality of the Computer Engineering discipline where the only that is constant is change.

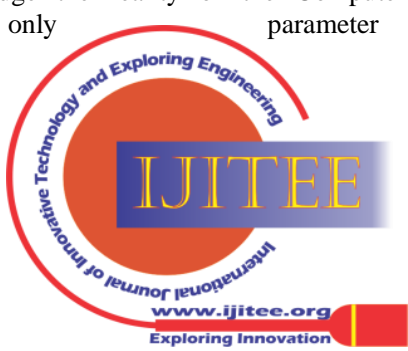

